\title{
Exploring Pre-University Students' Construction of Reasoned Argumentation during Computer - Supported Collaborative Discussions Using Sequential Analysis
}

\author{
Pavithra Panir Selvam and Aini Marina Ma'rof* \\ Faculty of Educational Studies, Universiti Putra Malaysia, 43400 UPM Serdang, Malaysia
}

\begin{abstract}
The prominent role of reasoning skills in predicting academic outcomes is clearly evident over the years in that its inculcation in various face-to-face learning contexts has become progressively dominant, including in the collaborative learning (CL) settings. The pandemic crisis, however, challenged traditional learning approaches to shift to an online mode overnight resulting in dramatic changes of learning delivery whereby teaching is undertaken remotely and on digital platforms. Though impact of CL-based approaches in promoting reasoning skills have been well-documented over the years, a systematic analysis of learners' behavioural patterns of argumentation and reasoning in a virtual collaborative learning environment is yet to be concretely established. The current study therefore sought to investigate the development of reasoned argumentation skills among pre- university students with mixed language abilities, using open-ending short stories via a computer-supported collaborative learning (CSCL) environment. Adopting the case study research design by applying a mixed-methods approach through both descriptive and sequential analyses, 12 pre-university students from a public research university served as participants of this study. The results show that language ability has a strong predictive factor on reasoned argumentation skills and there is an established tendency of the participants to produce constructive arguments over defensive or challenging viewpoints to alternative ideas. This calls for future studies to further investigate predictive factors of this tendency and to further ascertain the

ARTICLE INFO

Article history:

Received: 13 November 2020

Accepted: 11 March 2021

Published: 14 April 2021

DOI: https://doi.org/10.47836/pjssh.29.S1.17

$\overline{\text { E-mail addresses: }}$

pavithra@upm.edu.my (Pavithra Panir Selvam)

ainimarina@upm.edu.my (Aini Marina Ma'rof)

predictive role of language-rich discussions in facilitating various higher order thinking skills among learners.

Keywords: Collaborative reasoning, computersupported collaborative learning, reasoned argumentation skills, sequential analysis
\end{abstract}

* Corresponding author 


\section{INTRODUCTION}

Arguing is about putting forth one's disagreement; a general perception that has been speculated over a collaborative form of knowledge construction. The undeniable role of argumentation in information transfer and decision making has been studied over decades by researchers across disciplines. Argumentation is a social activity, rooting from different cultural and historical dimensions (Asterhan \& Schwarz, 2007) while reasoned argumentative practices have always been the start and endpoint of a discussion (Van Eemeren, 2017). Reasoning skills were found to be substantial, in various thought processes such as decision making and critical thinking (Chinn \& Samarapungavan, 2009). It enables the learner to formulate, learn and apply knowledge, promoting a better human civilisation. In today's VUCA world, the readiness to learn, unlearn and relearn, learning agility is the product of being able to reason progressively through continuous scaffolding processes. It is crucial for change agents such as teachers, policy makers and parents to anticipate and embrace the relevance of cultivating reasoned argumentative practices among learners. It has been the primary goal of the education system across the globe to equip learners to think critically and based on logical reasons, emerging from various viewpoints and conceptualize it according to the needs of the society. Reasoned argumentation has been highlighted as the key change in introducing an educational paradigm shift, ranging from primary to tertiary learning settings. The shift in the global education system needs has caused a total flip in the roles presented in a conventional classroom. Contrarily, there is a global consensus that students are easily bored and distracted by the oneway, teacher-dominated interaction largely present in today's classrooms. Students in these classrooms are merely receptors of the information provided to them and are not encouraged to contribute their own outof-the-box answers or questions.

It is important to retain students' attention by allowing them to participate voluntarily in learning sessions. Imposing gradual cognitive challenges through democratic discussions could cater to the growth of students' contextual knowledge, leading to an inclusive and a wholesome classroom interaction. A study on Malaysian students' critical thinking skills reveals that even after 11 years of schooling, the students failed to apply a proper reasoning on their judgements, be it in school or real world- related issues (Ghadi et al., 2013). This was further investigated through a larger study by Md Yunus et al. (2005) who found that the reasoning skills among the undergraduates from seven public universities were at a low moderate level, leaving a concern on the type of thinking workforce that we will produce in the coming decade. Reasoning skills should be taught in such a method that it is facilitated through exchange of ideas, feedback and by integrating application-related issues to learners. However, the current education scenario does not resemble such a learning environment and still focuses more on 
textbooks, seat-work and revision books (Anisah \& Delina, 2019). This will only allow for development of students' lower order thinking skills, neglecting the act of improvising their answers through argumentation and reasoning. Lee (2004) emphasised on how the Malaysian education system was still practising a didactic approach, using conventional exam-oriented pedagogies. This finding is supported by Ekwunife-Orakwue and Teng (2014) who argued that the implementation of such practices only focused on how to attain excellent grades instead of learning the content. In line with this, Darling-Hammond et al. (2020) argued that students were more driven to employ surface learning and study only to attain excellent grades rather than to engage in meaningful learning experiences and comprehend the subject matter, of which this defeated the actual purpose of education.

Karthikeyan (2017) characterised the current generation of learners as a society that lacked professional boundaries, influenced by socialization, demands an entitlement and lack of critical thinking skills. Collaborative learning (CL) in the form of argumentationbased approaches to learning alternatively promotes an in-depth understanding towards a specified content, giving a longer effect for learning gains to sustain (Nussbaum, 2008). To refine the umbrella term of CL, Anna et al. (2008) identified strategies in implementing an in depth argumentative discourse in classrooms through dialogic approaches. Collaborative reasoning (CR), is one educational dialogic approach that centralises on the dialogic inquiry as its main pedagogy that provides an open participation structure. Such an interactive discussion will encourage students to voice out their views and at the same time defend the views with proper reasoning as the views get opposed by others. Rogoff et al. (1995) proposed that learning took place during collaborative discussions as students were able to present their existing knowledge, and were competent enough to grow their ideas through fruitful peer interactions.

With the prevalence of the pandemic however, the computer-supported collaborative learning (CSCL) with the guide of advanced digital learning innovation has become progressively dominant in an assortment of instructional setting in supporting students' participation for authentic proof activities (Fatimah et al., 2020). The efficiency of such mediating agents has been extensively studied but a systematic analysis of learners' behavioural patterns of argumentation and reasoning in a virtual collaborative learning environment is yet to be concretely established. Therefore, this study intended to analyse the development of reasoned argumentation skills among pre- university students using the computer-supported collaborative learning (CSCL) approach and socioscientific issues (SSI). This contributes to addressing the role of discussion and critical thinking skills in increasing students' academic performance through a facilitated in-depth argumentation. Specifically, the current study sought to analyse the level of the participants' English proficiency prior 
to participating in the CSCL discourse, to explore the levels of the participants' reasoned argumentation during a fivesession CSCL discourse, to examine the relationships between the participants' English language proficiency and their reasoned argumentation levels in a fivesession CSCL discourse, and to explore the transition state of students' reasoned argumentation strategies in a five-session CSCL discourse.

\section{Argumentation Facilitates Critical Thinking Skills}

Argumentation has been considered as one of the foundational tools that constitutes social knowledge in a specified context, based on its nature as a dialogical approach (Resnick et al., 2017). The probabilities of students to co-construct their knowledge and refine their collective understanding towards a topic discussed are higher in an argumentative discourse compared to a static, one way information exchange, be it in an educational or a social setting (Felton et al., 2015). Based on the comparison, it was found that argumentation serves three (3) main purposes in fostering students' critical thinking competency; an exchange of competitive ideas, critical analysis on the given ideas, and the social arbitration of meaning to be assigned. Wolfe and Britt (2008) emphasized that the state of being focused in progressing an argument would eventually drive the students to suppress the opposing views that could possibly be brought up. Such a reaction is known to be a biased act upon a collective approval due to the lack of skills in critically examining the content and devising a persuasive argument deliverance. Argumentations strive to not only project a structure of conversational discussion but also to deliver the semantics behind it for the approval of its members therefore, the understanding should include a range of structure and meaning for apprehension (Walton, 2010). Being engaged in a complex and two-folded thinking process, students will associate several strategies to reinforce their views, understand the opposing ideas and identify the clashing ground points that need to be discussed in-depth for clarification. An instantaneous response to provide solid counter arguments in a discussion is a product of continuous practice of critical thinking skills, in the form of argumentation.

Berland (2011) pointed out a strong case on how argumentation facilitates learning and thinking skills in general, which included skills and conceptual gains. Researchers (Asterhan \& Schwarz 2007; Nussbaum \& Sinatra, 2003; Voss \& Wiley, 1997) have conducted systematic analyses upon the conceptual knowledge gains that students acquire via argumentative practices. All three (3) studies conclude that argumentation significantly improves conceptual knowledge by encapsulating critical thinking skills within various social and socio-cognitive processes. The need to distinguish the difference between generating an explanation and an argument centralises on the learner to be critical in retrieving information that suits the subjectmatter discussed. This includes minimising 
the amount of perceived risks (personal peer conflicts and fear to lose friendship), while executing a critical argumentative discussion (Tyson, 2011). Students do agree that argumentation and conflicts are helpful in understanding a topic in learning. However, they perceive disagreement as a lead to negative social consequences. Social risks are among the main predictors of why students end up supporting peer views and suppress their own (Bellmore, 2011). Disagreements in argumentative discussions pave ways to reflect upon an idea and to analyse misconceptions, thus promotes a conceptual change of ideas (Andriessen, 2006). A properly regulated, risk- minimised, and argumentative discussion is beneficial in the field of education due to the increasing level of social sensitivity (Aikins et al., 2005). Therefore, Tyson (2011) proposed technology- mediated discussions to eliminate personal conflicts and to only facilitate argumentative stances.

\section{Reasoning Predicts Academic Performance}

Being a thoughtful process, reasoning is crucial in an average individual's life routine and the pattern of it. Researchers (Lin et al, 2015; Lohman \& Lakin, 2009) have emphasized important skills as products of reasoning, problem-solving, decision making, critical thinking, meta-cognition and knowledge acquisition. Students with better reasoning abilities tend to draw more conclusions in a problem-solution relationship. A list of generalized reasoning skills was adapted by Bhat (2016) namely, inductive, deductive, linear, analogical, conditional and cause-and-effect, to be analysed within academic settings. The experiment explicitly imposed that reasoning skills could help students in understanding the underlying concepts of a situation and promote a rational-based approach towards it. Tella et al. (2008) claimed reasoning as one of the essential cognitive abilities that needed to be focused in assessing the impact of education on learning. A lesson that integrates reasoning within its structure, eventually assists students to gain knowledge based on logic and rationality. Having the required domain knowledge is essential in reasoning the initial conceptualization of a problem in order to understand the essential problem-solving strategy. This condition is vital when the acquired knowledge needs to be applied in a different situation and reasoning from various perspectives are required for approval.

\section{Collaborative Learning (CL) Enhances Discussion}

Collaborative learning (CL) refers to pedagogies and educational settings that foster cooperation among students in completing a common task where each individual is equally responsible and accountable for the task (Ekaterina \& Suzana, 2016). Collectively, students are assigned to seek meaning, relationships, inquiries, and decisions; redefining the conventional teacher- student roles in a classroom. Ekaterina and Suzana (2016) also outlined the significant educational and psychological contribution that 
CL could impose upon undergraduate education in three (3) main areas namely cognitive approach, social constructivism, and motivation. Students are found to learn best when they receive perspectives upon ideas, challenged for validation and assessed in a learning environment that provides both independence and interdependence. Lipman (2003) labeled CL as a platform that created a community of inquiry (CI) that was proficient in reasoning, discussing, challenging ideas, and deliberating. Researchers (Chandra, 2015; Lee \& Smagorinsky, 2000; Rogoff et al., 2003) have also indicated how engaging CL in classrooms could eventually aid students' academic achievement in certain areas. Looking into a socialinteraction based method, it is also crucial to address the possible concerns that can possibly be associated with it during the implementation. Peer facilitation also imposes significant positive impact towards students' academic achievement in various learning environments, such as blended learning (Lim et al., 2020a). CL takes place in a context, and this has rooted issues on both physical and contextual influences (culture, environment, and beliefs) that question the efficiency of CL in promoting collaborative discussions (Sadhana et al., 2011). Therefore, the need to have a tool that can mediate a collaboration, regardless of the contextual influences, were brought by both cognitivists and psychologists. Such self-regulative methods could facilitate students' online learning experience as per mentioned by Lim et al. (2020b). In conjunction with this recommendation,
Tyson (2011) proposed a technologymediated collaborative discussion which could avoid the elements such as personal conflicts and environmental influences.

The overarching objective of the study therefore, is to analyse the developmental patterns of reasoned argumentation strategies among pre-university students in a CSCL environment. Specifically, it sought to answer the following questions and hypotheses:

1. What is the level of participants' English proficiency prior to participating in CSCL discourse?

2. What is the level of participants' reasoned argumentation during a five-session CSCL discourse?

3. What is the relationship between participants' English proficiency and their reasoned argumentation level in a five-session CSCL discourse?

4. What is the transition state of students' reasoned argumentation strategies in a five- session CSCL discourse?

$\mathrm{H}_{1}$ : Level of language proficiency significantly predicts students' ability to produce claims in a CSCL discourse.

$\mathrm{H}_{2}$ : Level of language proficiency significantly predicts students' ability to produce supporting statements in a CSCL discourse.

$\mathrm{H}_{3}$ : Level of language proficiency significantly predicts students' ability to produce warrants in a CSCL discourse. 
$\mathrm{H}_{4}$ : Level of language proficiency significantly predicts students' ability to produce backing in a CSCL discourse.

$\mathrm{H}_{5}$ : Level of language proficiency significantly predicts students' ability to produce qualifiers in a CSCL discourse.

$\mathrm{H}_{6}$ : Level of language proficiency significantly predicts students' ability to produce rebuttals in a CSCL discourse.

\section{METHODS}

\section{Participants}

The population being studied in this research was pre-university students aged between 18-19 years old, from a Malaysian public research university that offers foundational studies. Based on the Malaysian education system, qualified high school leavers are required to complete a preparatory program before being eligible to pursue their studies at the bachelor's level. This particular group of population was selected in analysing reasoned argumentation because according to Hollingsworth and Rogers (2016), this is the phase where young adults start to consolidate their comprehension on abstract concepts, evaluate consequences and actively test their decision-making skills in an environment. Kremen et al. (2019) had pointed out that cognitive gains are rapid and highly adaptable at the average early adulthood of a learner. The age range that has been widely studied is from 18 to 20 years old. The study has also suggested the educational quality and cognitive reserves (thinking abilities) to be fostered by improving educational quality and firststage accessibility towards knowledge.

Quota sampling was carried out to identify the required participants, based on their availability for the study. The total population of the study was 913 foundation students, from both science and agricultural courses. The eligibility to participate in the study is that; 1) the participants should possess a minimal level of English language proficiency, for the discourses, 2) willing to commit throughout the five sessions being conducted, 3) aged between 18- 20 years old and 4) willing to intensively discuss and reflect openly about an issue. Twelve students were selected and divided into 3 groups; 4 in a group, assigned randomly. The sorting was made by their instructors based on their participation and performance in their English classes. The participants who volunteered to join the study were later shortlisted by their English language instructors based on their English language proficiency level, ranging from low, medium and high. The raw score was not available for the researcher to analyse due to the students' privacy protection reasons. The groups consisted of students from each category, as an approach to encourage having students of mixed abilities in every discussion. This is because CR is a structured approach that promotes an open participation among the participants, providing an opportunity to convert their monologues into dialogues within a group discussion (Reznitskaya, 2001). This composition was also expected 
to increase the involvement from students, regardless of their vocabulary limitations, self-confidence, and motivational level as per what has been outlined by Miller et al. (2014), in understanding the impact of $\mathrm{CR}$ and in facilitating active and reflective participation during CR discussions.

\section{Procedures}

CSCL Discussions. Based on a scheduled timing, participants were assembled through the researcher's Google Meet platform, a similar method that has been utilised for their primary learning session in classes. For 5 consecutive sessions, the participants were instructed to discuss and argue upon different short stories which embed real life problems that they might encounter in the future. The stories have an unresolved ending with two options of actions, which required the participants to argue and decide by the end of the discussion. Each group was given a maximum time of 20 minutes for every session to point out their stance and arguments and to sustain it. A total of 15 sessions, 3 groups with 5 sessions each, were recorded using the features available on the online platform. No particular role(s) was assigned to the participants in any of the discussion, leaving the researcher to take up the role of facilitating the discussions. Prompts were only provided if the discussion pauses for 10 seconds and above. An introduction and a conclusion must be made in every discussion, by both the participants and facilitator to reconfirm the decided side(s).
Post Research. The recordings of the discussions were then transcribed to be analysed according to the prepared coding scheme. Both content and sequential analysis were carried out to identify the pattern of reasoned argumentation among the participants. The results were then tabulated for analysis.

\section{MEASURES}

\section{Reasoned Argumentation}

Toulmin Model of Argument. In order to identify the usage of reasoned argumentation during a CSCL discourse, a content analysis was conducted on the transcribed discussion transcripts. The coding scheme applied in the research was based on the significant Toulmin model (1958), citing on Kulatunga and Lewis's (2013) work in studying both reasoned argumentation and the participation patterns in peer-led sessions. This coding illustrates the type of argument schema produced by the participants during the discussion through a thematic analysis. The coding scheme was divided into two main parts in the argument formulation, which were Creating (C) and Defending (D). Claim (CL) is the controlling idea of the proposed argument which relates directly to the statement to be proved. Support (SRT) is the evidence(s) that support the proposed claim; the strongest persuasive factor for the audience. Meanwhile, warrants (WRT) are assumptions or presumptions layering an argument. It is highly related to the belief/ culture/ societal beliefs upon a matter that caused the individual to claim a specific statement. The defensive skills are backing 
(BC), qualifier (QL) and rebuttal (RBT). $\mathrm{BC}$ is the proof(s) to solidify the attached warrant. QL are the expressions that reduce the certainty of having a prefixed assumption on a population. This addresses probability over the existing definite assumption(s). Lastly, RBT are the objections commonly made over arguments or supporting details that require further clarification. The first three skills are under $\mathrm{C}$ which indicate the tendency to formulate an idea and constantly enhancing it by evidence and social values in order to maintain a persuasive deliverance. In contrast, the additional three skills are meant to oppose and clarify opponents' viewpoints by raising confrontative questions during the CSCL discussion. Therefore, the later three skills were categorized as defending (D) in an argumentative discourse. The coding scheme served as the guideline to scrutinise the discussion transcripts, produced in all five CSCL discussions.

Open-ending Short Stories. The groups were presented with an incomplete short story to be discussed during every session. The task was to argue and collectively decide on the ending of the dilemma-based stories, at the end of each 20 minutes sessions. The decision with the majority vote from the team members would be assigned to the story. The stories revolve around possible authentic circumstances that the participants could encounter in their life or environment. Stories that challenge participants' moral beliefs and principles will establish an unbiased space for dynamic dialogic interaction to take place (Reznitskaya et al., 2009).
The content and suitability of the instrument was validated by two senior lecturers from Universiti Putra Malaysia. For reliability testing, two coders coded a total of 1107 stratagems from 5 continuous coding sessions. Stratagems are referred to the analysed argumentative aspects that were found in the participants' raw discussion transcripts. Sets is the term used to describe transcripts from every discussion and a sum of 15 sets were reviewed thoroughly for reliability. The transcripts were coded individually before it was compared between both coders. Reviewing the number of contrasting codes, both coders agreed upon 1103 out of 1107 stratagems that were found to be relevant. This resulted in a $99.81 \%$ of inter-coder agreement, and a Kappa value of 0.860 . According to Banerjee et al. (1999), a Kappa value ranging from 0.81 to 1.00 is almost a perfect agreement. Therefore, both coders had a good mutual understanding in interpreting the discussion transcripts.

\section{Data Analysis Strategy}

The data analyses for this study were collected through two different softwares, SPSS and GSEQ. SPSS was used to tabulate the descriptive statistics which are mean, range, standard deviation and logistics regressions involved in the study. This covers the language proficiency, reasoned argumentation levels and the relationship between the two variables using the Pearson Product Moment Coefficient. The regression analysis was used to examine the predicting factors of language competence on the participants' reasoned argumentation skills. 
GSEQ allowed the researcher to perform both content and sequential analyses at the group level. Content analysis has been deployed in order to define the behavioural interactions being presented through a dialogic deliverance. It was used to identify the most persistent argumentative strategy being applied by the participants in the CSCL discussions. Being a prominent method to understand sociological patterns, content analysis has its restrictions in examining the underlying relationship within the strings of coded messages.

These figures were then calculated within the sequential transition matrix calculation (Bakeman \& Gottman, 1997), illustrating the possibilities of the most commonly recurring argumentative behaviour in a discussion. This method is known as sequential analysis which requires an intercepting of a $\mathrm{z}$-score greater than 1.96 , between both row and column to be declared as significant ( $\mathrm{p}<0.05)$. Jeong et al. (2011) and Hou (2009) explained the efficiency of using sequential analysis in extracting the development of critical thinking skill within a discourse as it provided a deeper apprehension on the strategies being employed to solve a themespecific problem. The collected data are known as event sequential data (ESD) which segments the behavioural pattern in a single stream of codes, without any time factor being imposed upon. Prior to its realistic nature, this study applied the event recording scheme of the sequential analysis in collecting raw information from the argumentative discussion conducted. The tabulated final values were then analysed into a transition state diagram explaining different strategies developed and practiced by the participants in the CSCL discussions.

\section{RESULTS}

\section{Demographic Profile}

A total of 12 participants participated on a voluntary basis in the 5 -week study. The participants consisted of both international and local students who participated in the programme in the English course under different instructors. The participants' language proficiency levels that had been predetermined by their respective instructors were among the major information of the findings.

There were a total of $25 \%$ male participants $(n=3)$, followed by the remaining $75 \%$ of female participants $(n=$ 9). As for the participants' nationality, $33.33 \%$ were foreign students $(n=4)$ and $66.67 \%$ were local students $(n=8)$. The nationality differs as the pre-university/ foundation programme is offered on an international scale. The next major was their English language proficiency levels, which balanced equivalently with the $33.33 \%$ for all the categories; high, medium, and low. This composition was deliberately designed to ensure that every group was made up of different abilities in terms of language mastery. The distribution of their demographic details is illustrated in Table 1.

\section{Descriptive Analysis}

These analyses explain the first two research questions; a) the level of language 
proficiency prior to the participation in the CSCL discussions and b) the level of reasoned argumentation skills during the CSCL discussions. The range, mean, and standard deviation were tabulated to identify both the participants' language and

Table 1

Demographic profile of participants

\begin{tabular}{lcc}
\hline $\begin{array}{c}\text { Demographic } \\
\text { Profile }\end{array}$ & $\begin{array}{c}\text { Frequency } \\
(\mathbf{n}=\mathbf{1 2})\end{array}$ & $\begin{array}{c}\text { Percentage } \\
\mathbf{( \% )}\end{array}$ \\
\hline Gender & & \\
$\quad$ Male & 3 & 25 \\
$\quad$ Female & 9 & 75 \\
Total & $\mathbf{1 2}$ & $\mathbf{1 0 0}$ \\
Nationality & & \\
$\quad$ Local & 8 & 33.33 \\
$\quad$ International & 4 & 66.67 \\
Total & $\mathbf{1 2}$ & $\mathbf{1 0 0}$ \\
Proficiency level & & \\
$\quad$ High (3.00) & 4 & 33.33 \\
$\quad$ Medium (2.00) & 4 & 33.33 \\
$\quad$ Low (1.00) & 4 & 33.33 \\
Total & $\mathbf{1 2}$ & $\mathbf{1 0 0}$ \\
\hline
\end{tabular}

argumentative skills. These values range from weekly to a grand sum at the end of the fifth session.

The participants' English language proficiency level was indicated by their instructor as low, medium and high. The raw scores were unavailable for analysis due to the confidentiality principles practiced by the faculty's constitution. The categorical information was tabulated and presented as shown in Table 1. A value of range was assigned to every proficiency level recorded.

In order to answer the second research question, this study employed 12 respondents, for five consecutive weeks, consisting of a few elements, which stresses out on the comparison of range, mean and standard deviation. These figures describe the existing level of argumentative skills retained by the participants.

The participants' range of argumentations skills was assessed by a set of constructs, on a weekly basis. The average mean score and

Table 2

Example of weekly argumentative skills tabulation by construct

\begin{tabular}{cccccc}
\hline \multirow{2}{*}{ Participant } & \multicolumn{5}{c}{ Claim (CL) } \\
\cline { 2 - 5 } & Week 1 & Week 2 & Week 3 & Week 4 & Week 5 \\
\hline SA & Medium & Low & Medium & Low & Medium \\
FY & Medium & Medium & High & Medium & Medium \\
SL & Medium & Low & Medium & Medium & Low \\
NT & Medium & Low & Medium & Medium & Medium \\
AF & Medium & Low & Medium & Medium & Low \\
NS & High & Medium & High & High & Low \\
NA & High & High & High & Medium & Low \\
SR & Medium & Low & Medium & Medium & Medium \\
SS & Medium & Medium & High & Low & Medium \\
LS & High & Medium & High & Medium & Medium \\
AZ & Medium & Medium & Medium & Medium & Medium \\
AB & Medium & Low & Medium & Low & Medium \\
\hline
\end{tabular}


Table 3

Distribution of range for argumentation skills

\begin{tabular}{lccc}
\hline \multirow{2}{*}{\multicolumn{1}{c}{ Variables }} & \multicolumn{3}{c}{ Range } \\
\cline { 2 - 4 } Weekly & Low & Medium & High \\
Claim (CL) & $<2$ & $2-3$ & $>3$ \\
Support (SRT) & $<2$ & $2-8$ & $>8$ \\
Warrant (WRT) & $<1$ & $1-6$ & $>6$ \\
Backing (BC) & $<1$ & $1-4$ & $>4$ \\
Qualifier (QL) & $<1$ & $1-3$ & $>3$ \\
Rebuttal (RBT) & $<1$ & $1-4$ & $>4$ \\
Grand total & & & \\
Claim (CL) & $<9$ & $9-15$ & $>15$ \\
Support (SRT) & $<13$ & $13-39$ & $>39$ \\
Warrant (WRT) & $<7$ & $7-28$ & $>28$ \\
Backing (BC) & $<9$ & $9-17$ & $>17$ \\
Qualifier (QL) & $<3$ & $3-17$ & $>17$ \\
Rebuttal (RBT) & $<7$ & $7-20$ & $>20$ \\
\hline
\end{tabular}

Table 4

Total score for argumentative skills

\begin{tabular}{lccc}
\hline & N & Mean & Std. Deviation \\
\hline CLTotal & 12 & 12.0833 & 3.47611 \\
SRTTotal & 12 & 26 & 13.14257 \\
WRTTotal & 12 & 17.75 & 11.02167 \\
BCTotal & 12 & 13.1667 & 4.64823 \\
QLTotal & 12 & 10 & 7.66337 \\
RBTTotal & 12 & 13.25 & 6.91671 \\
\hline
\end{tabular}

standard deviation were then recorded as an overall value of the participants' level of the argumentative skills. Table 2 illustrates the example of the tabulation made for the 12 participants in identifying their levels of mastery for CL.

The average mean scores were assessed based on the analysed set of ranges, tabulated in Table 3, varying from weekly to the grand total basis. The number of mean scores produced by every participant determined their range of mastery for that particular argumentative skill.

The total values according to constructs were tabulated as exhibited in Table 4 for further analysis to answer the second research question.

\section{Regression Analysis}

A Poisson regression analysis was conducted to identify the argumentative skill(s) that is significantly predictive by language proficiency within the 5 CSCL discussions. Based on the fourth assumption of the Poisson regression, all dependent variables (DVs) have met the Poisson distribution for the results to be accepted. The observed and expected counts for all six DVs were found to be statistically insignificant to the Kolmogorov Smirnov tabulation results, indicating a high similarity to the Poisson distribution. The use of Poisson regression for this particular research objective has met the vital assumptions of the analysis method:

I. The six DVs were count data at which the frequency of every skill was recorded in every CSCL discussion, on a weekly basis,

II. Constitutes one or more IV. This study has only one IV which was the language proficiency of the participants,

III. Every observation was independent and there was no interdependent relationship between sessions,

IV. The distribution of counts follows a normal Poisson distribution. 
Table 5 discusses the skewed count measures obtained to understand the predictive correlation between the assigned variables.

The results indicate that there is a significant association between SRT $(\chi 2=$ $30.530, \mathrm{p}=.000)$, WRT $(\chi 2=.27 .651, \mathrm{p}=$ $.000), \mathrm{QL}(\chi 2=.18 .921, \mathrm{p}=.000), \mathrm{RBT}(\chi 2$ $=.13 .2500, \mathrm{p}=.000)$ and gender. Both $\mathrm{CL}$ $(\chi 2=.345, \mathrm{p}=.842)$ and BC $(\chi 2=.3 .964, \mathrm{p}$ $=.138)$ are deemed to have an insignificant affiliation with the proposed language ability factor.

It can be concluded that four out of six argumentative skills are significantly predictive by language proficiency. The four skills, SRT, WRT, QL and RBT, have a p-value of .000 to qualify them to be strongly significant to have a high probability of being determined by language proficiency. Both CL and BC have a p-value of .842 and .138 , respectively. As p $>.005$, these two variables are inconsequential to be determined by language proficiency. Therefore, language proficiency has the ability to predict $66.67 \%$ of the presented argumentative skills, implying the major role of language proficiency in fostering reasoned argumentation. The first null hypothesis was rejected as there is a significant relationship between the participants' English language proficiency and reasoned argumentation skills among pre-university students in the CSCL settings.

\section{Content Analysis}

Content analysis is used to weave chunks of information presented in a raw text or discussion through a thorough inspection for meaning and relativity towards a specific topic. Hence, in this study, the discussion transcripts were coded according to the Toulmin Model of Argumentation and analysed for discourse- based behavioural pattern(s).

A total of 1107 stratagems were found after several inter- coder sessions and the frequency of every argumentative aspect is shown in Table 6 below. It can be concluded that the participants have a remarkably high tendency to provide supporting details to consolidate their main idea in the discussion with a value of $29 \%$ in total. The rank is then followed by warrant, the values and belief held by the articulator, with $19 \%$ of the total number of discussions conducted. Qualifier that provides a probable condition in a circumstance, was the least performed throughout the study (11\%).

Table 5

Poisson regression analysis

\begin{tabular}{llccc}
\hline Variable & $\mathbf{N}$ & Mean $(\boldsymbol{\mu})$ & Wald Chi-Square $(\mathbf{X})$ & p-value \\
\hline CLTotal & 12 & 12.0833 & .345 & .842 \\
SRTTotal & 12 & 26.0000 & 30.530 & .000 \\
WRTTotal & 12 & 17.7500 & 27.651 & .000 \\
BCTotal & 12 & 13.1667 & 3.964 & .138 \\
QLTotal & 12 & 10.0000 & 18.921 & .000 \\
RBTTotal & 12 & 13.2500 & 15.773 & .000 \\
\hline
\end{tabular}


Table 6

Distribution of argumentative aspects during CSCL discussions

\begin{tabular}{lccccccc}
\hline \multirow{2}{*}{ Element } & \multicolumn{9}{c}{ Discussion } & \multicolumn{1}{c}{ Total } & \% \\
\cline { 2 - 8 } & $\mathbf{1}$ & $\mathbf{2}$ & $\mathbf{3}$ & $\mathbf{4}$ & $\mathbf{5}$ & & \\
\hline CLAIM & 34 & 22 & 41 & 26 & 22 & 145 & 13 \\
SUPPORT & 46 & 47 & 51 & 94 & 74 & 312 & 29 \\
WARRANT & 34 & 44 & 47 & 49 & 39 & 213 & 19 \\
BACKING & 31 & 33 & 32 & 46 & 16 & 158 & 14 \\
QUALIFIER & 18 & 33 & 29 & 26 & 14 & 120 & 11 \\
REBUTTAL & 21 & 28 & 28 & 54 & 28 & 159 & 14 \\
& & & & & & 1107 & 100 \\
\hline
\end{tabular}

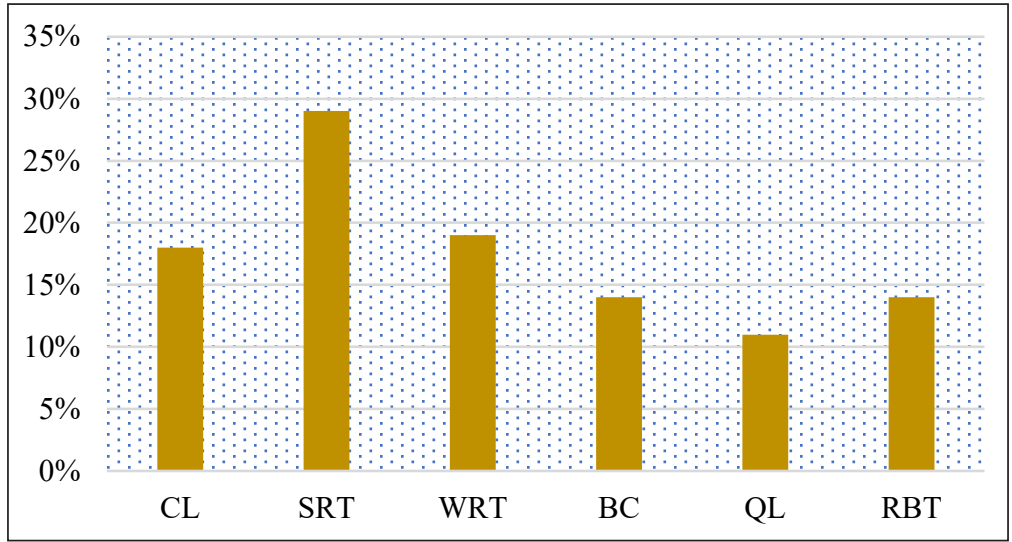

Figure 1. Distribution of argumentative aspects during CSCL discussions

According to the Toulmin Model of Argumentation, the findings have explained that the participants were all in the $\mathrm{C}$ category, whereby they were more concerned in building up strong arguments and refining them to be persuasive for the audience (Figure 1). The number of stratagems produced in the $\mathrm{C}$ category is higher in every discussion, resulting in a greater value in the grand sum from all the five CSCL discourses as shown in Table 7.

Therefore, the finding suggests that the participants were gravitating their thoughts in a constructive manner rather than confronting conflicting views to strengthen
Table 7

Group categorization based on total argumentative stratagems

\begin{tabular}{|c|c|c|c|}
\hline \multirow{2}{*}{ Group } & \multicolumn{2}{|c|}{ Argument Schema } & \multirow{2}{*}{$\begin{array}{c}\text { Group } \\
\text { Type }\end{array}$} \\
\hline & Creating & Defending & \\
\hline 1 & 130 & 109 & $\mathrm{C}$ \\
\hline 2 & 330 & 206 & $\mathrm{C}$ \\
\hline 3 & 195 & 137 & $\mathrm{C}$ \\
\hline
\end{tabular}

their allegation. The act of creating and enhancing an argument, holds up to $59.2 \%$ compared to challenging others' view(s) and reinforcing one's statement which constitutes a total of $40.2 \%$ of the entire data. More emphasis was placed on ensuring 
that their stand was properly articulated and persuasive enough, in contrast to identifying the clashing points within the ongoing discussion. The number of stratagems differs among groups. However the composition remains higher at the tendency of creating. For example;

NT: Others will be afraid to do this kind of shameless act, towards a child. AF: Truth is always the best policy because ermmm... even though that the police gather evidence from other people, they will take into account the past of the...suspects

The supporting statement from NT was not directly opposed by AF, but it was followed by a warrant and backing statements to strengthen her previous argument. Counter statements were given only when one was being challenged by more than one non-supporting participant.

\section{Sequential Analysis}

In order to understand the development of reasoned argumentation in every session, the sequential analysis was conducted based on the streams of coded stratagems produced. Table 8 illustrates an example of z- scores obtained from discussion 1, on all three groups that were present. Each row indicates the initial argumentative aspect while the column represents the follow- up strategy.

Table 9 represents the summary of z-scores obtained across the five CSCL discussions. The average was tabulated accordingly into a table of significance to explain the recurrence of strategies and the development of reasoned argumentation over the study period.

Table 8

Example of z-score for discussion 1

\begin{tabular}{lcccccc}
\hline $\mathbf{z}$ & $\mathbf{C L}$ & SRT & WRT & BC & QL & RBT \\
\hline CL & 0.55 & 3.87 & -2.10 & -1.23 & -1.49 & -0.52 \\
SRT & -0.85 & -3.23 & 3.38 & 2.30 & -0.25 & -1.17 \\
WRT & 0.55 & 0.31 & -2.10 & 1.91 & -1.49 & 0.68 \\
BC & 1.90 & -1.17 & 1.17 & -1.55 & -0.01 & -0.32 \\
QL & -2.09 & 1.42 & -0.27 & -1.29 & 0.16 & 2.23 \\
RBT & -0.46 & -0.68 & -0.59 & -0.88 & 3.78 & -0.33 \\
\hline
\end{tabular}

Table 9

Summary of z-scores across five CSCL discussions

\begin{tabular}{lcccccc}
\hline $\mathbf{z}$ & $\mathbf{C L}$ & SRT & WRT & BC & QL & RBT \\
\hline CL & 0.39 & 4.43 & -2.13 & -1.80 & -2.07 & 0.00 \\
SRT & -0.65 & -4.98 & 4.71 & 2.90 & 0.02 & -1.22 \\
WRT & 1.00 & 0.30 & -2.93 & 1.96 & -0.52 & 0.49 \\
BC & 0.77 & -2.24 & 2.07 & -1.92 & 1.30 & 0.54 \\
QL & -0.76 & 1.45 & -1.15 & -1.55 & 0.47 & 1.27 \\
RBT & -0.74 & 2.67 & -1.70 & -0.87 & 0.83 & -0.65 \\
\hline
\end{tabular}


The above scores were pooled for a sum and arranged according to the significant z- score obtained in every discussion. State transition diagrams were then constructed to illustrate the strategic flow within the weekly discussions. Table 10 ranks the significant sequences, according to the tabulated $z$ scores that were $>1.96 /(-1.96)$, that will be used to sketch the transitional diagram.

Discussion 1 and 2 have five significant sequences involving CL, SRT, WRT, QL, and RBT. This depicts that the first two discussions had a fair balance between creating an argument and defending the argument using an additional reinforcement(s). Discussion 3 had the least with only one significant sequence involving one element from the $\mathrm{C}$ and $\mathrm{D}$ categories, $\mathrm{CL}$ and RBT. The number increased gradually from the $4^{\text {th }}$ and $5^{\text {th }}$ discussion with 2 and 3 intercepting values. The zscore however is higher on formulating skills, SRT, WRT and CL, compared to destructive statements which are RBT, QL

Table 10

Overall significant sequence order

\begin{tabular}{lc}
\hline Significant Sequence & z- Score \\
\hline CL- SRT & 4.43 \\
SRT- WRT & 4.71 \\
WRT- BC & 1.96 \\
BC- WRT & 2.07 \\
RBT- SRT & 2.67 \\
SRT- BC & 2.9 \\
CL-WRT & -2.13 \\
CL- QL & -2.07 \\
SRT-SRT & -4.98 \\
WRT-WRT & -2.93 \\
BC-SRT & -2.24 \\
\hline
\end{tabular}

and BC. Comparisons were made between discussions and the developmental pattern of reasoned argumentation was interpreted in the five following Figures 2, 3, 4, 5, 6 .

From the above Figures 2, 3, 4, 5, 6, there are 16 significant sequences that can be found from all the five CSCL discussions. SRT constitutes the highest number of significances with 8 correlating patterns with other skills. This indicates that almost every participant in every discussion provided a minimum of 5-7 supporting details to project their thoughts. The rank is then followed by CL, WRT, $\mathrm{QL}$ and RBT with 4 significant sequences each, within the tabulated discussions. BC seems to have the least number of significant sequence with a total pattern of 4 recorded. It is interesting to note that the first two discussions have a strong salient sequencing between argumentative aspects involving all six sequences provided. The rate then starts to fluctuate on the following sessions, with reciprocating sequences among skills.

Week 1 has three significant sequences that have an extremely high z- score, CLSRT, SRT- WRT and RBT- QL, which means that the interactional pattern did not occur merely by chance. The probability of providing strongly refined arguments with several assumption- based objections can be seen at this particular week. Week 2 has a similar amount of significant sequences but within a different subset of skills which are, CL- SRT, QL- RBT and SRT- BC. This discussion has portrayed the groups to be in a mixed manner for reasoned argumentation whereby the discussion 


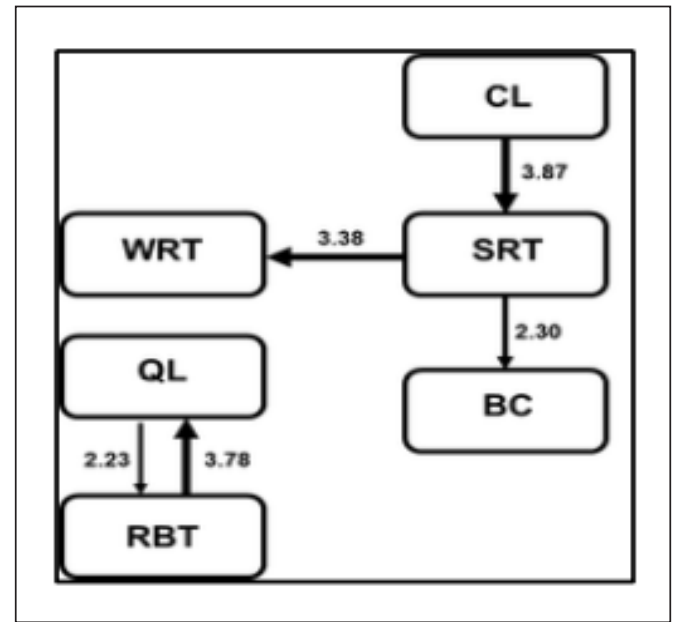

Figure 2. Discussion 1

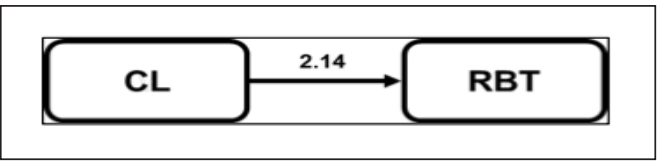

Figure 4. Discussion 3

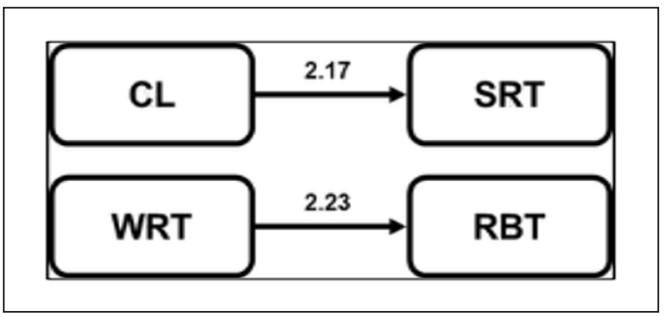

Figure 5. Discussion 4

took place in between providing supported statements followed by series of justified confrontation in the form of both questions and assumptions. Compared to other weeks, week 3 has the least significantly correlating pattern, which is only one. The paradigm shift among the participants is obvious whereby the most prominent method is opposing the main idea being put forth. The z- score explains that is moderately higher compared to any other discursive pattern's

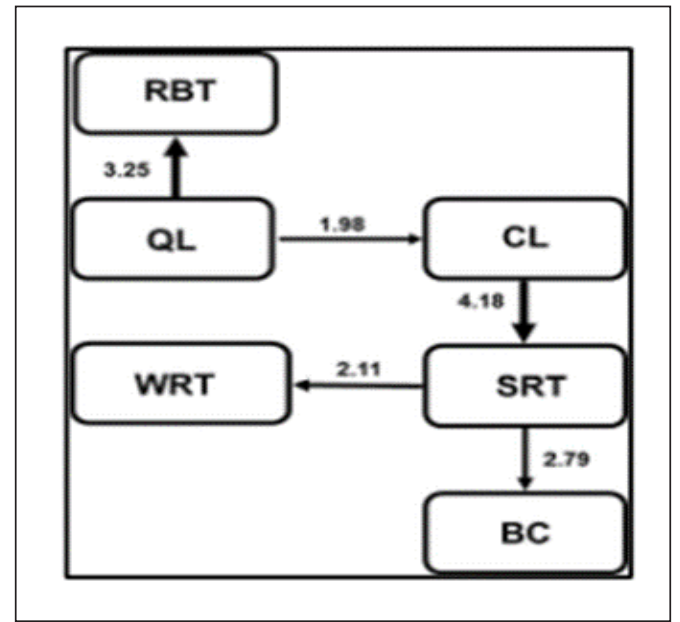

Figure 3. Discussion 2

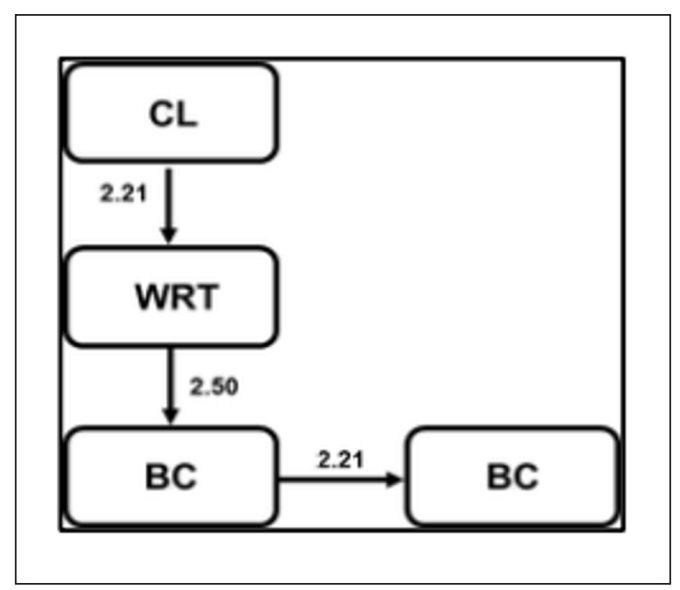

Figure 6. Discussion 5

present for the week, on average. Week 4 reflects the participants' nature to construct arguments rather than dispute viewpoints. Seventy-five percent (75\%) of the recorded significant sequence are among the first three argumentative skills which have the propensity towards building statements and not disrupting. The WRT- RBT signifies that the stated values and beliefs were the commonly questioned element in the weekly discussion, the short story about conflicts 
between offering help and holding a grudge. The final discussion enhanced the previous reasoned argumentation behaviour among the participants, having three significant sequences, SRT- WRT, WRT- BC, as well as $\mathrm{BC}$ and $\mathrm{QL}$, emphasizing on a constructive discourse.

Figure 7 exemplifies the average significant sequences that were recorded throughout the study period. This provides the decision on the third hypothesis of the study by analysing the overall transitional state of the participants' reasoned argumentation. There are six significant sequences that can be derived as the final product, involving five of the aspects outlined in the coding scheme. Two sequences with the highest z- score would be CL- SRT and SRTWRT, strongly predicts the argumentative behaviour of generating arguments. This is followed by SRT- BC and RBT- SRT, adding to the idea of constructive CSCL discussions with a reasonable number of objections and clarifications provided by the participants. Lastly, WRT- BC has the cut off $z$ - score

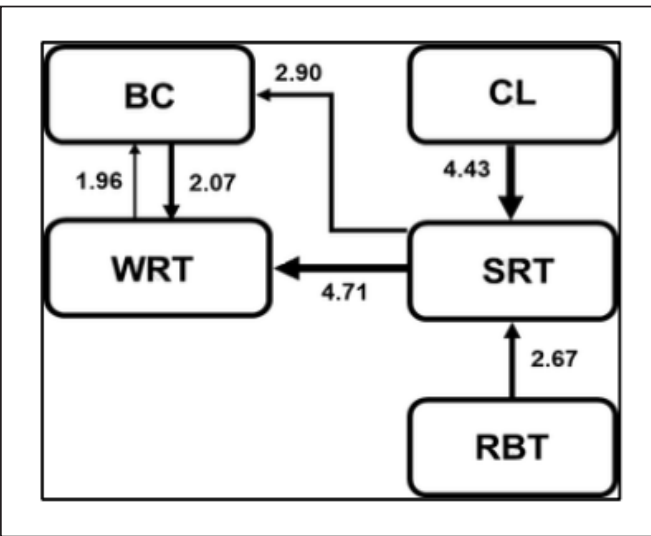

Figure 7. Final developmental pattern of reasoned argumentation across five CSCL discussions of 1.96, showing that there is an enough number of validating examples given to strengthen several values being enunciated. QL was found to be insignificant to the other aspects with only one sequence and having a $z$ - score of -2.07 . The sequence was not included as the $\mathrm{z}$ - score is $<1.96$. Therefore, the second null hypothesis was rejected as there is a significant state of transition in students' reasoned argumentation strategies throughout the five-session CSCL discourse.

\section{DISCUSSION}

Objective 1: To Analyse the Level of Participants' English Proficiency prior to Participating in the CSCL Discourse

The English language proficiency of the participants was pre-determined by the instructors based on the participants' recent test scores. The composition of every group was balanced with one participant from each proficiency level (low, medium, and high). The decision of having mixed abilities in each group summed the general idea of ensuring a successful CR setting for the collaborative discourse to take place. The purpose was to understand the role of language proficiency and how it affects reasoned argumentation skills.

Objective 2: To Explore the Level of Participants' Reasoned Argumentation during a Five-session the CSCL Discourse

Based on both the descriptive and content analyses carried out, it was evident that the participants exhibited constructive argumentative skills more than half of the 
time. Providing claim, support and warrant occupied the overall stratagem distribution by $13 \%(\mu=12.0833), 29 \%(\mu=26)$ and $19 \%$ $(\mu=17.75)$, respectively. At this pace, the participants performed a steady tendency to be aware in structuring their statements to be convincing for the rest of the group members. All three groups were classified within the $\mathrm{C}$ category which entitles to a strong acknowledgement for content compared to being at variance towards external thoughts.

\section{Objective 3: To Examine the Relationship between the Participants' English Language Proficiency and their Reasoned Argumentation Level in a Five-session CSCL Discourse}

A Poisson regression analysis was carried out to scrutinize the possible argumentative skills that can be predicted by one's language proficiency. It was crystal clear that language has a remarkably high influence in predicting more than half of the discourse aspects; four out of six.

\section{Objective 4: To Explore the Transition State of the Students' Reasoned Argumentation Strategies in a Five- session CSCL Discourse}

The sequential analysis indicates the probable and recurring reasoned argumentation strategies appropriated throughout the CSCL discussions. The skills with the highest z-scores and correlated significantly are from the creating category $(\mathrm{C})$ of the devised coding scheme. This confirms the previous information tabulated from the content analysis on the participants' level of reasoned argumentation. This study has provided an explicit evidence on several practical concerns for the attention of educators, which will all benefit the learners at large.

\section{Predicting the Role of Language Proficiency in Developing Critical Thinking Skills}

Zhang and Doughtery Stahl (2011) had outlined the key requirements in ensuring a successful collaborative learning environment and language comprehension of which this was one of the listed criteria. Objectives 1 to 3 emphasised on outlining the level and predictive strength of language proficiency towards the analysed argumentative skills. It is evident that language acts is one of the prominent tools in facilitating reasoned argumentation among the participants. In this study, students with a better language acquisition managed to articulate the ideas and refute clearly in the focused group discussion while those with less proficiency struggled to properly enunciate their thoughts. This view has been validated by Paul (2004) that a good language ability is imperative in establishing a good foundation for critical thinking skills among young adult learners. The significance of embedding language improvement through communicative approaches within the education system provides an opportunity for learners to enhance their ability to assume, generate ideas and reflect on the language (Owen 
et al., 2019). The developmental role of language proficiency was later addressed by Grosser and Nel (2013), of which they discovered an interdependent correlation between language mastery and application of critical thinking skills. The fact that learners or individuals mostly process their thoughts in their first language and the possible effects it could imply upon in a social interaction that requires a second language, is continuously studied by social psychologists. Jordan and Johan (2015) implied that the act of 'dismantling' language from the thought was impossible, therefore, the point of mentioning how it influences the variables are merely meaningless. This point was scientifically endorsed by Hassan Taj et al. (2017) of which their study portrays how a broad domain of language at a normal range could significantly impact a learner's cognition in terms of semantic and syntactic processing. Hence, projecting an integral argument or statement requires a competency in the language in the social event that is taking place. This is ambivalent in the quality of participation that could affect the overall reasoning process that one would like to express in a discourse.

Stakeholders, educators, policy makers and education- based industries, should start looking into the effort of integrating language-rich discussions into students' learning experiences. Familiarising the learners to speak and think in a different language enables them to elevate their awareness to think critically in any circumstances. This relates to the point clarified by Ghadi et al. (2013) that language does not only enhance linguistic features but also activate a learner's capacity to relate and engage mentally in the current real- life issues. Therefore, there is a dire need to ensure the language proficiency of students to foster better critical thinking skills.

\section{Reasoned Argumentation Facilitates Knowledge Sustainability}

The study has explained that every group performed differently with a significant argumentation strategy in every discussion. Although the interactions were constructive in nature (CL, SRT, and WRT) they lacked verbal reinforcing argumentative techniques (BC, QL, and RBT). The participants' priority was given to agreeing to provide statements, elaborating self- generated leads and justifying their choices. Based on the findings of the content analysis, the participants tended to settle more frequently in creating a viewpoint and were less inclined to challenge other views. Such conservative reactions in a discussion can be caused by many factors, embodied within the environment of the articulator.

Maeda (2017) elucidated that selfefficacy, dealing with fear, judgement, isolation and discrimination, as among the prominent factors that affected the rate of classroom discussion. Learners tend to be anxious about the public view of him/ her when a challenging attempt is made towards a perspective. Hajar et al. (2017) had also explained the consequences of this socialacceptance phenomenon within the Social Networks Analysis (SNA) perspective. 
This notion was further analysed by Uphill et al. (2014) on how such an adverse emotional state could cause an inability in decision making, increase the amount of poor learning involvement and academic dissatisfaction. The mentioned issues are a part of the argumentative skills deficiency theory, which aims to alleviate the unnecessary verbally abusive behaviour due to the lack of appropriate knowledge on the manners of delivering a disagreement upon a subject matter.

Collaborative argumentation is one of the practically suggested methods in handling an inactivity in a classroom discussion. Within this spectrum, learners will be able to propose a claim, support a side, weight arguments, refute challenging views, reflect on the rationality, and decide upon a solution/party (Jiménez-Aleixandre, 2007). Task-based learning and collaborative learning should be encouraged among young adult learners as they benefit better in an active session that provides feedback, challenges, and justifications rather than a monologue or one-way learning approach. This suggestion is supported by several previous researchers who advocate the idea of having more than just information as the key factor in determining a successful knowledge construction as well as sustainment (Weinberger, 2011). Tan and Wong (2020) had also indicated the need for learners to learn beyond the syllabus, within an ICT- integrated learning environment, in order to adapt to a futuristic learning approach.

\section{LIMITATIONS AND RECOMMENDATIONS}

The generalisation of the results is subjected to a certain population, provided that their learning environment and age group are pre-determined. The participants should possess at least a moderate level of English language mastery to be able to participate in CSCL discussions. The intensity of the discussion might differ depending on several fundamental factors related to the participants, such as age, general knowledge, and language competency. Besides, the temporality of data collected in a CSCL setting is another major concern that restricts the generalisation of the findings. As CSCL discourses take place over a period of time, a large number of episodes should be studied to refine the understanding of the effects of the setup towards reasoned argumentation.

Future researchers could broaden the participant's perspectives by studying a different age and geographical cultural groups. As this study has addressed the significant role of language in predicting argumentative skills, upcoming studies could conduct comparative assessments on the impact of different language proficiency levels upon learners' argumentation levels. This can be an added value that can consolidate language proficiency as a determining factor in nurturing higher order thinking skills. Language-rich activities, interactive speech-related competitions, and public speaking are some of the activities that can be focused on in future studies. 


\section{CONCLUSIONS}

To conclude, this study has used several statistical measures to identify the relationship and developmental strategies between language proficiency and argumentation skills. The participants' argumentation level data were tabulated to be compared over the sessions and total data. The participants exhibited a higher tendency to construct their arguments and concretize the arguments with the necessary supporting details (CL, SRT, and WRT) rather than confronting a conflicting viewpoint (BC, QL, and RBT) during the CSCL discussions. It had also been found that language proficiency was a strong predictor of reasoned argumentation skills throughout the argumentative discourse. Reasoned argumentation should be fostered in the current education system that is facing the evolving VUCA world due to the Covid-19 pandemic, which demands an extremely high level of reasoning before deciding on a matter.

The responsibility of ensuring a widespread awareness should not be shouldered by the Ministry of Education and school teachers only, but also by the entire community in order to foresee and understand the advantages proposed by a simple and yet analytical approach in the education paradigm.

\section{ACKNOWLEDGEMENT}

We would like to acknowledge to the respondents who were willing to participate and provide full cooperation in this study.

\section{REFERENCES}

Aikins, J. W., Bierman, K. L., \& Parker, J. G. (2005). Navigating the transition to junior high school: The influence of pre-transition friendship and self-system characteristics. Social Development, 14(1), 42-60. doi: 10.1111/j.14679507.2005.00290.x

Andriessen, J. (2006). Arguing to learn. In K. Sawyer (Ed.), Handbook of the learning sciences (pp. 443-459). Cambridge University Press.

Anisah, I. M., \& Delina, M. (2019). Augmented reality water rocket: Develop an enrichment book of physics. Journal of Physics: Conference Series, 1185(1), 012044. doi:10.1088/1742$6596 / 1185 / 1 / 012044$

Asterhan, C. S. C. \& Schwarz, B. B. (2007). The effects of dialogical and monological argumentation on concept learning in evolutionary theory. Journal of Educational Psychology, 99(3), 626-639. doi: 10.1037/0022-0663.99.3.626

Bakeman, R., \& Gottman, J. M. (1997). Observing interaction: An introduction to sequential analysis. (2nd Ed.). Cambridge University Press

Banerjee, M., Capozzoli, M., McSweeney, L. \& Sinha, D. (1999). Beyond kappa: A review of interrater agreement measures. Canadian Journal of Statistics, 27, 3-23. doi: 10.2307/3315487

Bellmore, A. (2011). Peer rejection and unpopularity: Associations with GPAs across the transition to middle school. Journal of Educational Psychology, 103(2), 282-295. doi: 10.1037/ a0023312

Berland, L. K. (2011). Explaining variation in how classroom communities adapt the practice of scientific argumentation. Journal of the Learning Sciences, 20(4), 625-664. doi: $10.1080 / 10508406.2011 .591718$

Bhat, M. (2016). The predictive power of reasoning ability on academic achievement. International 
Journal of Learning, Teaching and Educational Research, 15(1), 79-88.

Chandra, R. (2015). Collaborative learning for educational achievement. IOSR Journal of Research \& Method in Education (IOSRJRME), 5(3), 2320-7388. doi: 10.9790/738805342629

Chinn, C. A., \& Samarapungavan, A. L. A. (2009). Conceptual change-multiple routes, multiple mechanisms: A commentary on Ohlsson (2009). Educational Psychologist, 44(1), 4857. doi: 10.1080/00461520802616291

Darling-Hammond, L., Flook, L., CookHarvey, C., Barron, B., \& Osher, D. (2020). Implications for educational practice of the science of learning and development. Applied Developmental Science, 24(2), 97-140. doi: 10.1080/10888691.2018.1537791

Ekaterina G. \& Suzana S. (2016). Basic principles of "Collaborative Learning". European Scientific Journal, 17(2011). doi: 10.19044/esj.2011. $\mathrm{v} 17 \mathrm{n} 0 \mathrm{p} \% 25 \mathrm{p}$

Ekwunife-Orakwue, K. C., \& Teng, T. L. (2014). The impact of transactional distance dialogic interactions on student learning outcomes in online and blended environments. Computers \& Education, 78, 414-427. doi: 10.1016/j. compedu.2014.06.011

Fatimah, F., Rajiani, S., \& Abbas, E. (2020). Cultural and individual characteristics in adopting computer-supported collaborative learning during covid-19 outbreak: Willingness or obligatory to accept technology? Management Science Letters, 11(2), 373-378. doi: 10.5267/j. msl.2020.9.032

Felton, M., Garcia-Mila, M., Villarroel, C., \& Gilabert, S. (2015). Arguing collaboratively: Argumentative discourse types and their potential for knowledge building. British Journal of Educational Psychology, 85(3), 372-386. doi: 10.1111/bjep. 12078

Ghadi, I. N., Bakar, K. A., Alwi, N. H., \& Talib, O. (2013). Measuring critical thinking skills of undergraduate students in Universiti Putra Malaysia. International Journal of Asian Social Science, 3(6), 1458-1466.

Grosser, M. M., \& Nel, M. (2013). The relationship between the critical thinking skills and the academic language proficiency of prospective teachers. South African journal of education, 33(2). doi: 10.15700/saje.v33n2a639

Hou, H. T. (2009). A framework for dynamic sequential behavioral pattern detecting and automatic feedback/guidance designing for online discussion learning environments. Raquel Hijon-Neira, IntechOpen.

Jeong, A., Clark, D. B., Sampson, V. D., \& Menekse, M. (2011). Sequential analysis of scientific argumentation in asynchronous online discussion environments. In Puntambekar, S., Erkens, G., \& Hmelo-Silver, C. (Eds), Analyzing interactions in CSCL (pp. 207-233). Springer.

Jiménez-Aleixandre, M. P. (2007). Designing argumentation learning environments. In Sibel, E. \& Jiménez-Aleixandre, M. P. (Eds.), Argumentation in science education (pp. 91115). Springer.

Karthikeyan, C. (2017). A qualitative study on managing millennial mindsets: Awaiting challenges for HR strategists and recruiters. International Journal of Innovative Research in Engineering \& Management, 4(1), 601-609. doi: 10.21276/ijirem.2017.4.1.10

Kremen, W. S., Beck, A., Elman, J. A., Gustavson, D. E., Reynolds, C. A., Tu, X. M., SandersonCimino, M. E., Panizzon, M. S., Vuoksimaa, E., Toomey, R., Nennema-Notestine, C., Hagler Jr., D. J., Fang, B., Dale, A. M., Lyons, M. J., \& 
Franz, C. E. (2019). Influence of young adult cognitive ability and additional education on later-life cognition. Proceedings of the National Academy of Sciences, 116(6), 2021-2026. doi: 10.1073/pnas.1811537116

Kulatunga, U., \& Lewis, J. E. (2013). Exploration of peer leader verbal behaviors as they intervene with small groups in college general chemistry. Chemistry Education Research and Practice, 14(4), 576-588. doi: 10.1039/C3RP00081H

Lee, M. N. (2004). Global trends, national policies and institutional responses: Restructuring higher education in Malaysia. Educational Research for Policy and Practice, 3(1), 31-46. doi: 10.1007/ s10671-004-6034-y

Lee, C. D. \& Smagorinsky, P. (2000). Vygotskian perspectives on literacy research: Constructing meaning through collaborative inquiry. Cambridge University Press.

Lim, C., Ab Jalil, H., Ma'rof, A., \& Saad, W. (2020a). Peer learning, self-regulated learning and academic achievement in blended learning courses: A structural equation modeling approach. International Journal of Emerging Technologies in Learning (iJET), 15(3), 110-125. doi: 10.3991/ijet.v15i03.12031

Lim, C. L., Ab Jalil, H., Ma'rof, A. M., \& Saad, W. Z. (2020b). Self-regulated learning as a mediator in the relationship between peer learning and online learning satisfaction: A study of a private university in Malaysia. Malaysian Journal of Learning and Instruction, 17(1), 51-75.

Lin, T. J., Anderson, R. C., Jadallah, M., NguyenJahiel, K., Kim, I. H., Kuo, L. J., Miller, B. W., Logis, H. A., Dong, T., Wu, X., \& Li, Y. (2015). Social influences on children's development of relational thinking during smallgroup discussions. Contemporary Educational Psychology, 41, 83-97. doi: 10.1016/j. cedpsych.2014.12.004
Lipman, M. (2003). Thinking in education (2nd Ed.). Cambridge University Press.

Lohman, D. F., \& Lakin, J. M. (2009). Reasoning and intelligence. In R. Sternberg, \& S. Kaufman, Handbook of intelligence (2nd Ed.). Cambridge University Press.

Maeda, J. (2017). Self-efficacy reduces impediments to classroom discussion for international students: Fear, embarrassment, social isolation, judgment, and discrimination. IAFOR Journal of Education, 5(2), 141-159. doi: 10.22492/ ije.5.2.07

Md Yunus, A. S., Abu, R., Nor, S. M., Tarmizi, R. A., Bakar, K. A., Ali, W. Z. W., Hamzah, R., \& Ismail, H. (2005). Generic skills of Malaysian university students. Bulletin of Higher Education Research. Instititut Penyelidikan Pendidikan Tinggi Negara, (6), 5-6.

Miller, B. W., Anderson, R. C., Morris, J., Lin, T. J., Jadallah, M., \& Sun, J. (2014). The effects of reading to prepare for argumentative discussion on cognitive engagement and conceptual growth. Learning and Instruction, 33, 67-80. doi: 10.1016/j.learninstruc.2014.04.003

Nussbaum, E. M., \& Sinatra, G. M. (2003). Argument and conceptual engagement. Contemporary Educational Psychology, 28, 384-395. doi: 10.1016/S0361-476X(02)00038-3

Nussbaum, E. M. (2008). Collaborative discourse, argumentation, and learning: Preface and literature review. Contemporary Educational Psychology, 33, 345-359. doi: 10.1016/j. cedpsych.2008.06.001

Owen, E. A., Razali, A. B., Samad, A. A., \& Noordin, N. (2019). Enhancing Libyan students' English speaking performance through language game and information gap activities. Problems of Education in the 21st Century, 77(1), 110. doi: $10.33225 / \mathrm{pec} / 19.77 .110$ 
Paul, R. W. (2004). Critical thinking: What every person needs to survive in a rapidly changing world. Sonomata State University, Centre for Critical Thinking.

Resnick, I., Davatzes, A., Newcombe, N. S., \& Shipley, T. F. (2017). Using relational reasoning to learn about scientific phenomena at unfamiliar scales. Educational Psychology Review, 29(1), 11-25. doi: 10.1007/s10648-016-9371-5

Reznitskaya, A., Anderson, R. C., McNurlen, B., Nguyen-Jahiel, K., Archodidou, A., \& Kim, S. (2001). Influence of oral discussion on written argument. Discourse Processes, 32(2\&3), 155-175. doi: 10.1080/0163853X.2001.9651596

Reznitskaya, A., Kuo, L. J., Clark, A. M., Miller, B., Jadallah, M., Anderson, R. C., \& Nguyen-Jahiel, K. (2009). Collaborative reasoning: A dialogic approach to group discussions. Cambridge journal of education, 39(1), 29-48. doi: $10.1080 / 03057640802701952$

Rogoff, B., Baker-Sennett, J., Lacasa, P., \& Goldsmith, D. (1995). Development through participation in sociocultural activity. New Directions for Child and Adolescent Development, 1995(67), 45-65. doi: 10.1002/cd.23219956707

Rogoff, B., Paradise, R., Arauz, R. M., CorreaChavez, M., \& Angelillo, C. (2003). Firsthand learning through intent participation. Annual review of psychology, 54(1), 175-203. doi: 10.1146/annurev.psych.54.101601.145118

Hassan Taj, I., Ali, F., Sipra, M., \& Ahmad, W. (2017). Effect of technology enhanced language learning on vocabulary acquisition of EFL learners. International Journal of Applied Linguistics \& English Literature, 6(3), 11. doi:10.7575/aiac.ijalel.v.6n.3p.262

Tan, B. S., \& Wong, S. L. (2020). Learning principles of accounting in ICT-supported learning environments of Malaysian secondary schools:
Future-oriented approach. Research and Practice in Technology Enhanced Learning, 15(1), 1-23. doi: 10.1186/s41039-020-00128-6

Tella, A., Tella, A., Adika, L. O., \& Toyobo, M. O. (2008). Relationship among demographic variables and pupils' reasoning ability. Electronic Journal of Research in Educational Psychology, 6(3), $709-728$.

Tyson, W. (2011). Modeling engineering degree attainment using high school and college physics and calculus course taking and achievement. Journal of Engineering Education, 100(4), 760777. doi: 10.1002/j.2168-9830.2011.tb00035.x

Uphill, M., Groom, R., \& Jones, M. (2014). The influence of in-game emotions on basketball performance. European Journal of Sport Science, 14(1), 76-83. doi: $10.1080 / 17461391.2012 .729088$

Van Eemeren, F. H. (2017). Argumentative patterns viewed from a pragma-dialectical perspective: Exploring the relationship between argumentative discourse and institutional context. In Prototypical argumentative patterns. (pp. 7-29). John Benjamins Publishing.

Voss, J. F., \& Wiley, J. (1997). Developing understanding while writing essays in history. International Journal of Educational Research, 27(3), 255-265. doi: 10.1016/S08830355(97)89733-9

Walton, D. (2010). Burden of proof in deliberation dialogs. In P. McBurney, I. Rahwan, S. Parsons \& N. Maudet (Eds.), Argumentation in multi-agent systems (pp. 1-22). Springer

Weinberger, D. (2011). Too big to know: Rethinking knowledge now that the facts aren't the facts, experts are everywhere, and the smartest person in the room is the room. Basic Books.

Wolfe, C. R. \& Britt, M. A. (2008). The locus of the myside bias in written argumentation. 
Pavithra Panir Selvam and Aini Marina Ma'rof

Thinking \& Reasoning, 14,1-27. doi: 10.1080/13546780701527674

Zhang, J. \& Doughtery Stahl, K. A. (2011). Collaborative reasoning: Language-rich discussions for English learners. The Reading Teacher 65(4), 257-260. doi: 10.1002/ TRTR.01040 